\begin{tabular}{|c|c|c|c|}
\hline Eiszeitalter u.Gegenwart & $\mathbf{3 0}$ & $\begin{array}{c}183-201 \\
11 \mathrm{Abb} .\end{array}$ & Hannover 1980 \\
\hline
\end{tabular}

\title{
Computerauswertung von Seespiegeldaten für das IGCP-Projekt Nr. 61
}

\author{
Horst Preuss *) \\ Sea level, data processing, computer, radiometric dating, Holocene \\ Northsea, North West German Lowlands
}

\begin{abstract}
Kurzfassung: Ein Teil des deutschen Beitrages zum IGCP Sea Level Project war die Computerauswertung von Seespiegeldaten. Dies umfaßte den Entwurf eines Formblattes für die Datenerfassung sowie die Entwicklung eines Computerprogrammsystems zur Auswertung und Verarbeitung der Daten bis zu graphischen Zeit-/Tiefen-Diagrammen für verschiedene Meeresspiegelstände innerhalb der letzten 10000 Jahre.

Zur Verarbeitung vorgesehen sind alle Daten, die einen Zeit-/Tiefen-Bezug zum Meeresspiegel haben, vorwiegend jedoch solche von radiometrisch datierten Meeresspiegel-Indikatoren. Besonders hervorgehoben wird die Möglichkeit der Umrechnung der zumeist unterschiedlichen Basisdaten auf eine einheitliche Bewertungsgrundlage, so daß direkte Vergleiche zwischen den einzelnen Zeit-/ Tiefen-Diagrammen von verschiedenen Küstenregionen und gegründet auf Daten der verschiedensten Labors erlaubt sind. Mehrere Beispiele von Umrechnungen und Darstellungen in Form von Diagrammen sind dieser Publikation beigegeben.
\end{abstract}

\section{[Computer Evaluation of Sea Level Data for IGCP-Project No. 61]}

A b s t r a c $t$ : Part of the German contribution to the IGCP Sea Level Project was the computer evaluation of sea level data. This included the development of a form for the data collection as well as the development of a set of computer programs for evaluating and retrieving data for display in time/depth graphs for various positions of the sea level within the last 10.000 years.

It is planned to evaluate all data which have a time/depth relation to sea level, but especially those of radiometrically dated sea level indicators. The possibility for converting the basic data into a homogeneous form to permit direct comparison of individual time/depth graphs of various coastal regions and data from different laboratories is especially emphasised. Several examples for transformations and representations in graphs are included in this publication.

$$
\text { Inhaltsverzeichnis }
$$

1. Einleitung

2. Konzeption eines Computerprogrammsystems

3. Datenerfassung und Formblattentwicklung

4. Datenverarbeitung mit Listen- und Plotterausgang

5. Schriftenverzeichnis

\section{Einleitung}

Das Projekt Nr. 61 des Internationalen Geologischen Korrelationsprogrammes (IGCP) mit dem Titel „Sea Level Changes During the last Deglacial Hemicycle“ wird auf nationaler Ebene seit 1975 von der Deutschen Forschungsgemeinschaft (DFG) gefördert.

Ein deutscher Beitrag zu dem internationalen Projekt war dabei der Versuch, Seespiegeldaten mit Hilfe der Computerverarbeitung (Arbeitsgruppe Preuss, Streif, Vinken, NLfB Hannover) auszuwerten.

*) Anschrift des Verfassers: Dr. H. Pre us s, Niedersächsisches Landesamt für Bodenforschung, Stilleweg 2, D-3000 Hannover 51. 


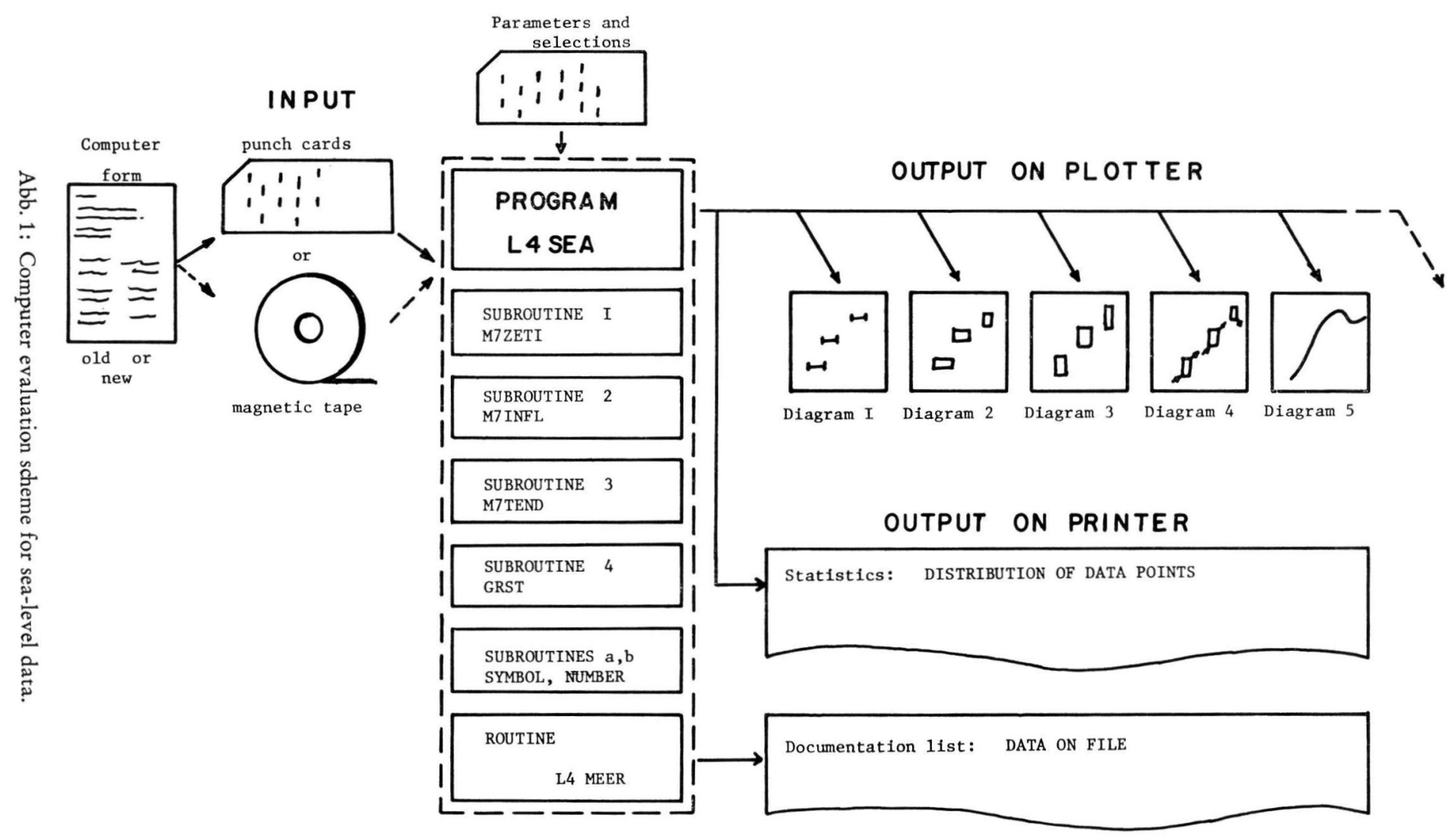


Der Ansatz hierzu wurde erstmals während eines Treffens einer Gruppe von Teilnehmern am IGCP-Projekt Nr. 61 in Haarlem (Niederlande) im Jahre 1975 diskutiert. Die Teilnehmer waren sich der großen Mengen von global gesammelten Meeresspiegeldaten bewußt und erkannten die Probleme, die in der Heterogenität der Daten liegen. An eine Computerverarbeitung dieser Daten wurden damals die Hoffnungen geknüpft, die zwingende Einheitlichkeit bei der Auswertung und Darstellung zu erreichen und dadurch direkte Vergleiche zu ermöglichen.

Viele der publizierten Meeresspiegelanstiegskurven von verschiedenen Küstenbereichen zeigen große Unterschiede im Inhalt und in der Konstruktion. Die Anwendung eines Computerprogramms zur Auswertung der Basisdaten erscheint sinnvoll, um individuelle Konstruktions-Eigenheiten zu vermeiden. Außerdem wird die testweise Anwendung verschiedener Modelle oder Modellvorstellungen zum Meeresspiegelanstieg und seinen steuernden Faktoren ermöglicht. Hier sind Geschwindigkeit und die Wiederholbarkeit der Rechenvorgänge mit veränderten Parametern von entscheidender Bedeutung.

Das Ziel, eine eustatische Meeresspiegelanstiegskurve von globaler Aussagekraft zu gewinnen, schien durch Faktoranalyse und Vergleiche der Regionalkurven erreichbar, so daß der Aufwand der Erfassung von Basisdaten in computerverarbeitbarer Form als lohnend betrachtet werden kann. Die einzelnen Schritte von der Konzeption eines Computerprogrammsystems, der Erfassung von Basisdaten, ihre Aufbereitung und Verarbeitung sowie ihre Ausgabe und graphische Darstellung sollen im folgenden nachgezeichnet werden.

\section{Konzeption eines Computerprogrammsystems}

Ausgehend von den oben beschriebenen Aufgabenstellungen wurde die Konzeption eines Programmsystems mit der Kurzbezeichnung L4 SEA entwickelt. Dieses Konzept umfaßt die Daten-Erfassung, -Verarbeitung und -Ausgabe (vgl. Abb. 1).

Die Eingabe sollte so variabel gehalten sein, daß sowohl Lochkartenstanzer als auch Bildschirmterminals als Datenerfassungsgeräte dienen können. Die Verarbeitung beinhaltet verschiedene Routinen zur Datenprüfung, -Angleichung, Fehlerberechnung, Umkodierung, Umrechnung, Modellberechnung, Datenauswahl und Steuerung der Ausgabe. Die Datenausgabe umfaßt sowohl die Druckerausgabe von Texten als auch die Plotterausgabe für graphische Darstellungen von Zeit/Tiefen-Diagrammen.

\section{Datenerfassung und Formblattentwicklung}

Um Klarheit darüber zu gewinnen, welche Informationen als Basisdaten dem Computerprogramm zu übergeben sind, mußten zunächst die Darstellungswünsche analysiert werden. Die hieraus entwickelten Vorschläge wurden mehrfach in internationalen Gesprächsrunden diskutiert, umgestellt und erweitert. Als erstes Ergebnis erschien Ende 1976 ein Computer-Formblatt zur Aufnahme aller derjenigen Daten, die sich auf einen Probeentnahmepunkt und die Beschreibung der dazugehörenden analysierten und datierten Probe beziehen.

Dies sind im einzelnen:

Geographische Lokalität des Probeentnahmepunktes, gemessene Position der Probe, geotektonische Einflüsse auf den Probenentnahmepunkt (soweit bestimmbar), Probenmaterial, Beprobungsmethode, ${ }^{14} \mathrm{C}$-Datierung oder eine andere Datierung mit Ergebnis, mögliche Kontamination, Beziehung der Probe zu einem fossilen Seespiegel, paläogeographische Situation, einige aktuelle Meeresspiegeldaten. 


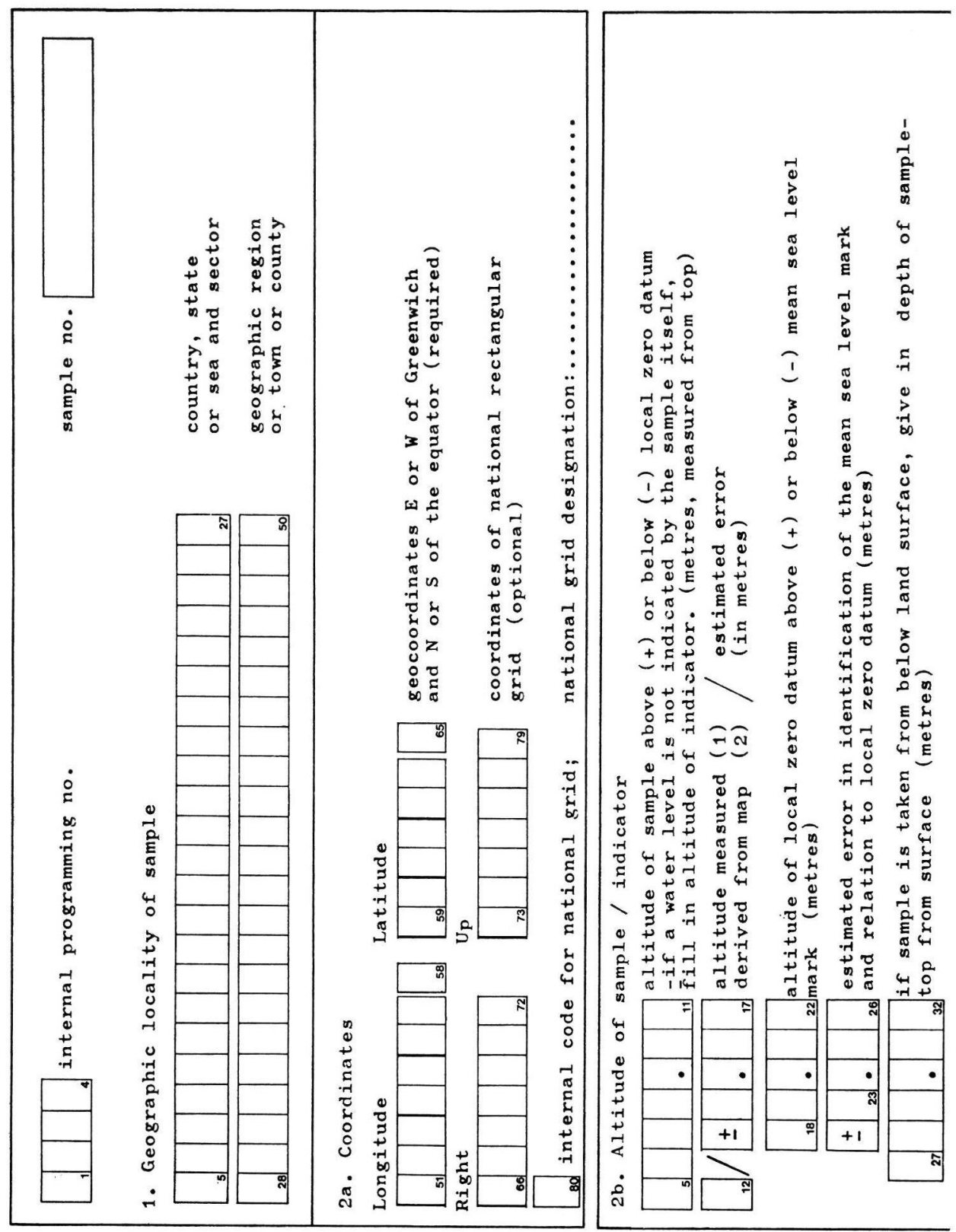

Abb. 2: Computer-Formblatt (2. Ausgabe, Frontseite).

Dieses Computer-Formblatt zeigte noch einige Mängel, so daß es, nachdem die 1. Auflage von 2000 Exemplaren vergriffen war, nicht mehr in der gleichen Form neu aufgelegt wurde. Mit dem Fortschritt der Programmierarbeiten sind mehrere Änderungen notwendig geworden, die Grundlage für die Diskussion um eine neue Auflage während einer 


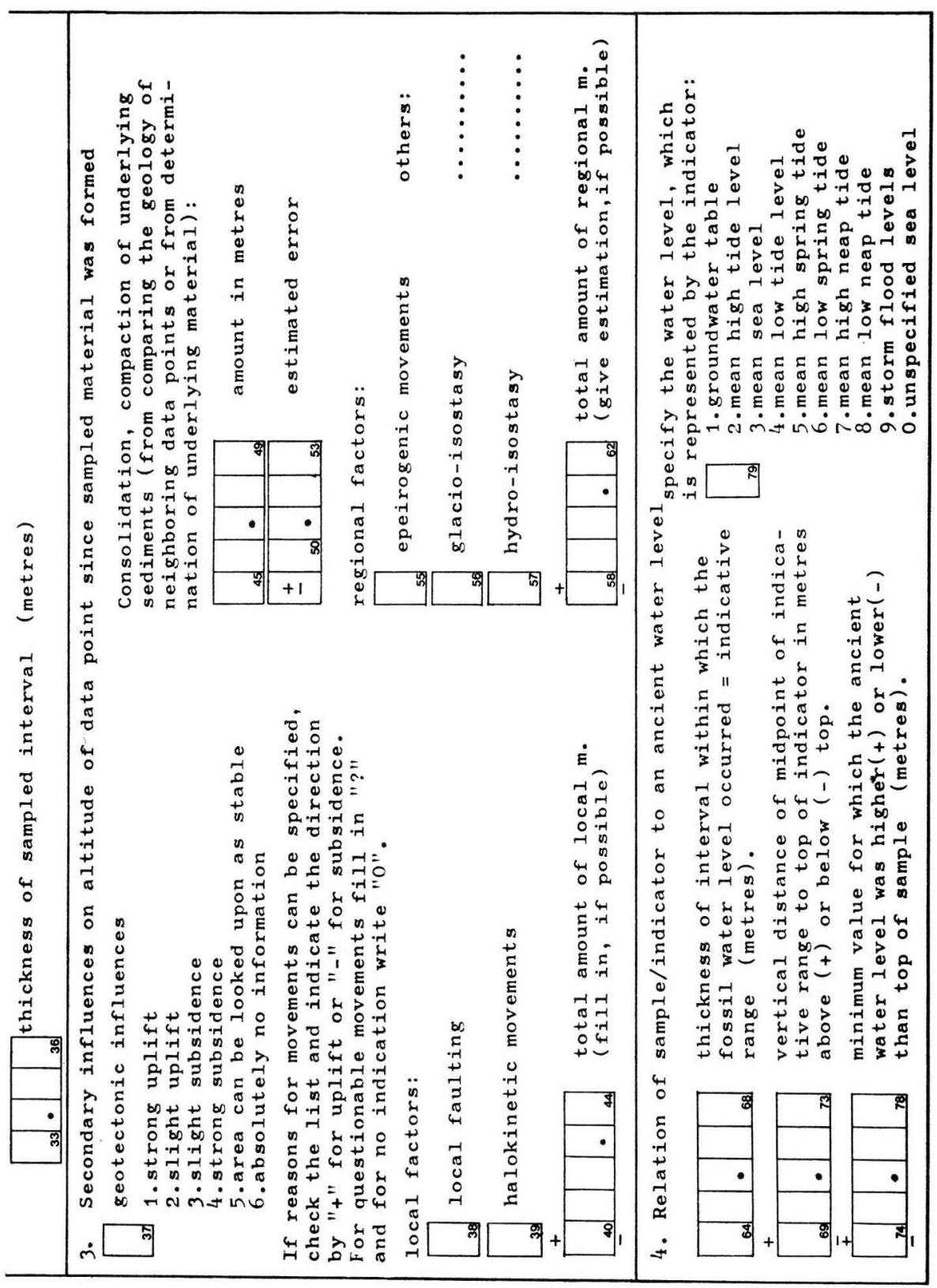

Arbeitstagung in Birmingham (England) vom 14.-24. August 1977 waren. Der Auftrag zur Neuentwicklung erging an die Hannoversche Arbeitsgruppe, die Unterstützung von der holländischen Arbeitsgruppe (Dr. O. v. D. Plassche) erhielt.

Die Erläuterungen zum Formblatt, die sog. „Explanatory Guidelines“, sind in mehreren Arbeitssitzungen in Hannover zusammen mit der niederländischen Arbeitsgruppe entwickelt worden. Sie sollen dem Formblattbenutzer das Ausfüllen erleichtern, indem 


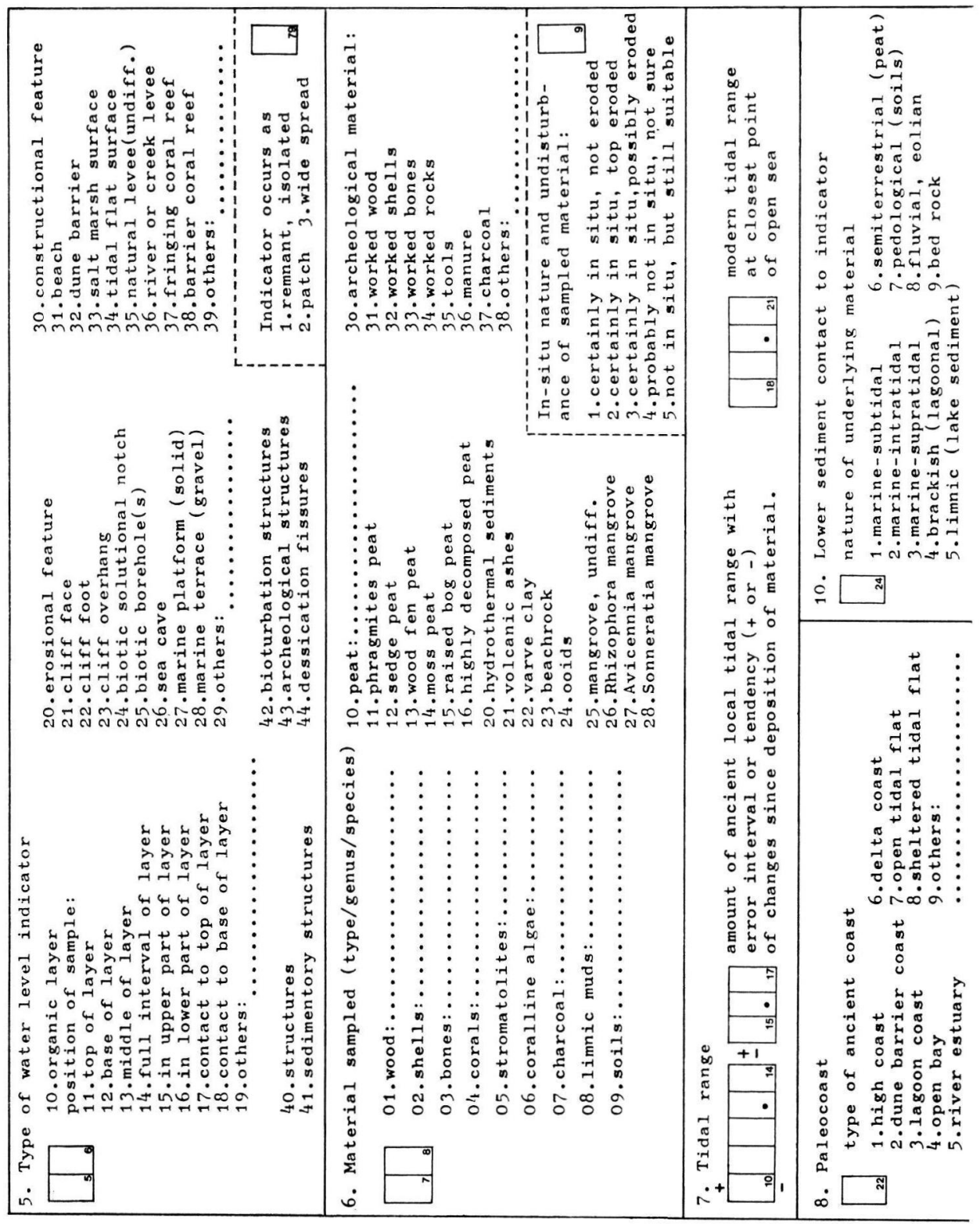

Abb. 3: Computer-Formblatt (2. Ausgabe, Rückseite).

sie verschiedene Möglichkeiten aufzeigen und Beispiele geben. Die Abbildungen 2 und 3 zeigen die zweite erweiterte Auflage des Formblattes, das in ca. 3000 Exemplaren an die Regionalvertreter des internationalen Projektes versandt worden ist. Weitere 1000 Formblätter lagen außerdem während der Tagung der Projektmitglieder in Sao Paulo (Brasilien) vom 11. bis 19. September 1978 zur Selbstbedienung aus. 


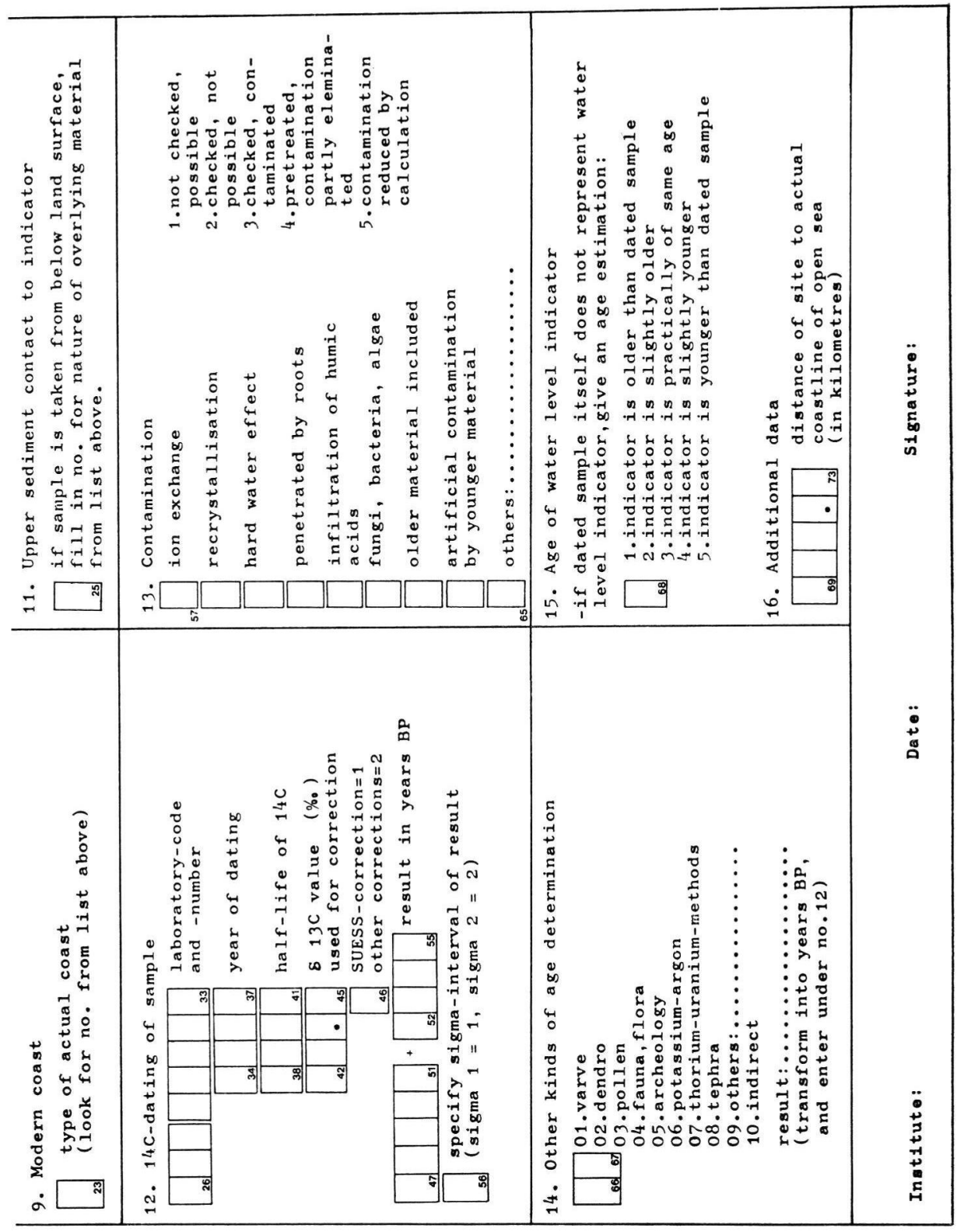

Die niedersächsischen Meeresspiegel-Basisdaten von datierten Proben, die im Rahmen des Projektes seit 1975 den Holozänprofilen im Küstenraum entnommen worden sind, sind zum größten Teil direkt auf das neue Formblatt aufgenommen. Nur zum kleineren Teil waren sie schon vor 1977 im alten Formblatt erfaßt worden und mußten umgeschrieben werden, um die Dateneingabe einheitlich zu gestalten.

Die alten vor 1975 im Rahmen der Geologischen Kartierung der Küstenblätter angefallenen Daten konnten teilweise aus dem Archiv des Niedersächsischen Landesamtes 


\section{$\begin{array}{clll}\text { LONGITUDE LATITUDE } & \text { R16HT } & \text { UP } \\ 0 & 0 & 3467645 & 5925600\end{array}$}

ALTITUDE OF SAMPLE TO ZERO DATUM: -8.19 $1=A L T I T U D E$ MEASURE $0,2=D E D U C E D$ FROM MAP: ESTIMATED ERROR OF ALTITUDE: $\quad 0.20 \mathrm{M}$ ALT ITUDE OF ZERO DATUM TO MSL: $11.57 \mathrm{M}$ $\begin{array}{ll}\text { IDENTIFICATION ERROR FOR MSL-MARK: } & 0.00 \mathrm{M} \\ \text { DEPTH OF SAMPLE BELOW SURFACE: } & 9.69 \mathrm{M}\end{array}$ THICKNESS OF SAMPLED INTERVAL: $0.05 \mathrm{M}$

RELATION OF SAMPLE TO AN ANCIENT W.L. SPECIFIED WATER LEVEL:

SPECIFIED WATER LEVEL:

$2=M E A N$ HIGH TIDE LEVEL

$3=$ MEAN SEA LEVEL

$4=M E A N$ LOW TIDE LEVEL

S=MEAN HIGH SPRING TIDE

0 ( )

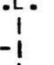

INFLUENCES ON ALTITUDE OF DATA POINT

GEOTECTONIC INFLUENCES:

3

$1=$ STRONG UPLIF T

$2=S L I G H T$ UPLIFT
$3=S L I G H T$ SUBSIDENCE

$3=$ SLIGHT SUBSIDENCE
$4=S T R O N G$ SUBSI DENCE

$5=\angle O O K E D$ UPON AS STABLE

$5=$ LO KED UPON AS
$6=N O$ INFORMATION

CONSOLIDATION, COMPACTION

AMOUNT IN METRES: 0.50

AMOUNT IN METRES: 0.50

NATIONAL GRID CODE

LOCAL FACTORS

LOCAL FAULTING: 0

HALTKINETIC MOVES:

REGIONAL FACTORS

EPEIROGENIC MOVES:

GLAC10-1SOSTASY:

TOTAL AMOUNT: -0.8

OTHERS :

$7=$ MEAN HIGH NEAP TIDE

$8=$ ME AN LOW NEAP TIDE

$9=S T O R M$ FLOOD LEVEL

I $=$ UNSPECIFIED SEA LEVEL

FOSSIL WATER INTERVAL (INDICATIVE RANGE): $0.00 \mathrm{M}$ ISTANCE OF MIDPOINT TO TOP OF INDICATOR: MIN IMUM VALUE FOR HHICH THE ANCIENT HOL WAS HIGHER(+) OR LOHER(-) THAN SAMPLETOP:

TYPE OF WATER LEVEL INDICATOR: $10=L$.AYER

$10=L$. AYER
POSITION OF SAMPLE:

$11=$ TOP OF LAYER

$13=$ MIDDLE OF LAYER

14=FULL INTERVAL OF LAYER

$15=$ IN UPPER PART OF LAYER

$16=$ IN LOWER PART OF LAYER

$17=$ CONTACT TO TOP OF LAYER

I 18=CONTACT TO BASE OF LAYER

$20=E R O S I O N A L$ FEATURE

$21=C L I F F$ FACE

$22=$ CLIFF FOOT

$23=$ CLIFF OVERHANG

$4=B I O T I C$ SOLUTIONAL NOTCH

$5=R I O T I C$ BCREHOLE (S)

$26=S E A$ CAVE

I $19=0$ THERS

(MARINE PLATFORM (SOLID) $29=0$ THERS

$30=$ CONSTRUCTIONAL FEATURE

\section{$31=B E A C H$}

$33=S A L T$ MARSH SURFACE

$34=$ TIDAL FLAT SURFACE

35= NATURAL LEVEE (UNDIF. )

$36=R$ IVER OR (REEK LEVEE

$37=F R I N G I N G$ CORAL REEF

$39=0$ THERS
$40=S T R U C T U R E S$

41= SED IMENT ORY STRUCTURES

$42=B I$ OTURBAT ION STRUCTURES

$43=A R C H E O L O G I C A L$ STRUCTURES

$44=$ DESS ICATION FISSURES

INDICATOR OCCURS AS: 0

$1=1 S$ OLATED REMNANT

$2=P A T C H$

$3=W I D E$ SPREAD
MATERIAL SAMPLED: 10

I $O 1=W O O D$

$02=S H E L L S$

$103=B O N E S$

$04=$ CORALS

O5 = STROMATOLITES

O6=CORALLINE ALGAE

$07=$ CHARCOAL

08=LIMNIC MUDS

09= SOILS

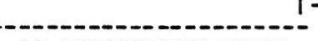
$10=$ PEATCUNSPECIFIED) $11=$ PHRAGMITES PEAT $12=$ SEDGE PEAT 13=FENWOOD PEAT $14=$ MOSS PEAT $15=$ R AISE D $80 G$ 16 = DECOMPOSED PEAT

\section{$20=$ HYDROTHERMAL SEDIM.} $21=$ VOLCANIC ASHE $22=V A R V E$ CLAY $23=$ EEACHR
$24=00$ IDS $25=$ MANGROVE (UNDIF.) $26=R$ HI ZOPHORA MANG $27=$ AVICENNIA MANG.
$28=$ SONNERATIA MANG.
$30=A R C H E O L O G$ IC MAT .

$31=$ WOR KED WOOD

$32=$ WORKED SHELLS

$33=$ WORKED BONES

$34=W$ ORKED ROCK

$35=$ TOOLS

$36=$ MANURE
$37=$ CHARCOAL $38=0$ THERS
IN-SITU NATURE: 0

I $1=I N-S I T U$, NOT ERODED

$12=I N-S I T U$, TOP ERODED

$3="$ POOSSIBLY ERODED

I $5=$ NOT IN-SITU
ANCIENT TIDAL RANGE:

$0.0 \# 0.0$

MODERN TIDAL RANGE:

3.6

PALEOCOAST:

MODERN COAST: 5

$1=H I G H$ COAST

$2=B A R R I E R$ COAST
$3=\angle A G O O N$ COAST

$4=O P E N$ BAY

$4=0 P E N$ BAY
$5=R I V E R$ ESTUARY

6=DELTA COAST

$7=O P E N$ TIDAL FLAT

$8=$ SHELTERED T.FL. 0

$07=$ TH OR IUM/URANIUM

NATURE OF UNDERLYING MAT:
NATURE OF OVERLYING MAT:

OTHER KINDS OF AGE DETERMINATION

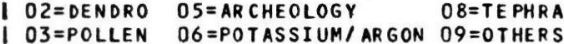

Distance of SITE TO ACTUAL COASTLINE:

$8.9 \mathrm{kM}$

AGE OF WATER LEVEL IND: 0

$2=S L$. OLDER $4=S L$. YOUNGER

$3=$ SAME AGE S=YOUNGER
14C-DATING OF SAMPLE:

LABORATORY AND NUMBER: HV 7193 YEAR OF DATING : HALF LIFE OF 14C (YEARS): 5570 $1=S U E S S$ CORRECTION $2=0$ THERS 26.9 $1=S U E S S$ CORRECTION, $2=0$ THERS: RESULT IN YEARS BP: $4800 \# 60$ CONTAMINAT ION:

$1=$ POS SIBLE

$2=N O T$ POSSIBLE $3=$ CONTAMINATED

$\begin{array}{ll}2=\text { NOT POSSIBLE } & 3=\text { CONTAMINATED } \\ 4=\text { PRETREATED } & 5=\text { CALCULATED }\end{array}$

ION: O ROOTS:O OLD MAT: 0

RECRYST: 0 ACIDS:0 YOUNG MAT:0 NOD
HARD WAT: 0 FUNGI:0 OTHERS: 00100 
für Bodenforschung übernommen werden, sofern ihre Ubbernahme nicht durch die Rechte der Einsender gesperrt war. Diese Daten sind zum größten Teil unvollständig und müssen bei der Auswertung zwangsläufig zur Darstellung größerer Fehlerintervalle führen. Dennoch sind sie als Stützwerte bei der Kurvenkonstruktion von großer Bedeutung. Der Versuch, fehlende Angaben (z. B. zum Sediment bzw. Fazieskontakt zur beprobten Schicht) in den Fachabteilungen des Landesamtes oder außerhalb des Landesamtes zu suchen und zu ergänzen, war bislang nur bedingt erfolgreich, und der Aufwand für solche Ergänzungen ist in dem Falle erheblich. Bis zum gegenwärtigen Zeitpunkt existieren mehr als 800 ausgefüllte Formblätter mit Daten von Proben aus den niedersächsischen Küstengebieten. Davon sind bislang 519 auf Magnetband abgespeichert worden.

\section{Datenverarbeitung mit Listen- und Plotterausgang}

Ein Programm zur Auslistung der kompletten auf Datenträger übernommenen Daten wurde bereits Ende 1976 entwickelt (Abb. 4).

Wahlweise ließen sich Schnelldruckerlisten mit den Daten zur geographischen Lokalität und gemessenen Positionen der Proben (= Kopfdaten) erstellen. Außerdem konnten Plotbilder auf dem Schnelldrucker erzeugt werden, die die Daten als Sterne oder als Großbuchstaben (Abkürzungen für ihre Indikation) im Zeit/Tiefen-Diagramm unverändert wiedergaben (Dokumentation). Hier ist zu erwähnen, daß Schnelldruckerplotbilder keine Detaildarstellungen erlauben, sondern nur einen groben Úberblick geben können. Es war deshalb nicht möglich, Fehlerintervalle darzustellen (siehe Abb. 5).

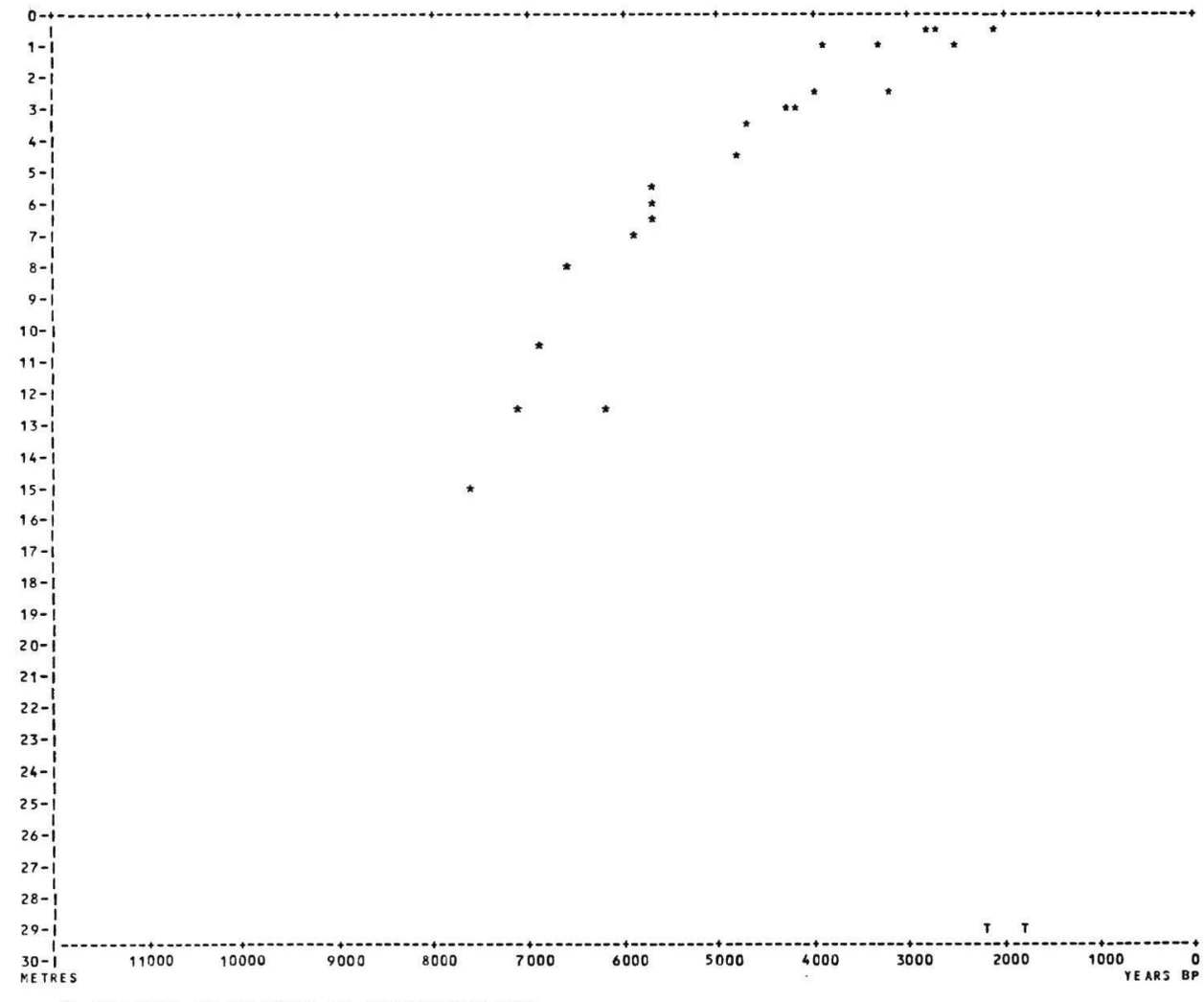

26 DATA FORNS RRE EVALUATED FOR DOCUMENTATION PLOT

Abb. 5: Zeit/Tiefen-Diagramm als Plotbild. 
IGCP PROJECT N0.61: SEA-LEVEL CHANGES

STATISTICAL DISPLAY OF SEA-LEVEL DATA:

USA. DE. LEWES CREEK PROVIDES

USA. NJ. MAURICE RIVER PROVIDES

USA. DE, ISLAND FIELD PROVIDES

USA. NJ. NANTUXENT

PROVIOES

USA, DE, AUGUSTINE

PROVIDES

USA. MD, ASSATEAGUE IS. PROVIDES

USA. DE, HOLLY OAK PROVIDES

USA, VA, CHINCOTEAGUE PROVIDES

USA. DE, PEPPER CREEK PROVIDES

FRANCE, CANCALE-ILLE PROVIDES

RYUKYU ISLANDS, OKINAWA PROVIDES

FRANCE, MEDITERRANEAN PROVIOES

NEW CALEDONIA, CENTRAL PROVIOES

INDONESIA, JEPARA, JAVA PROVIOES

SPAIN, CAMPO DE DALIAS PROVIOES

MAURITANIE, AFTOUT ES PROVIDES

SENEGAL. MBODIENE PETIT PROVIOES

MOROCCO, HAHA

PROVIDES

BENIN (EX-DAHOMEY)

PROVIOES

USA. CT, CLINTON, haMmu PROVIDES

USA, CLINTON, CT PROVIDES

BRAZIL, SAO-PAULO PROVIDES

IVORY COAST, WEST AFRIK PROVIDES

GREECE, GYTHION

PROVIOES
33 LATA POINTS (OLO FURMAT) 1 DATA POINTS (OLD FORMAT) 16 DATA POINTS (OLD FORMAT) 1 DATA POINTS (OLD FORMAT) 6 DATA POINTS (OLD FORAAT) 1 DATA POINTS (OLD FORMAT) 12 OATA POINTS (OLO FORMAT) 1 DATA POINTS IOLO FURMATI 22 DATA POINIS (OLD FORMAT) 4 DATA POINTS (OLD FORMAT) 2 DATA POINTS (OLD FORMAT) 2 DATA POINTS (OLD FORAAT) 6 DATA POINTS (OLO FORMAI) a DATA POINIS IOLD FORMATI 1 DATA POINTS (OLD FORMAT) 2 OATA POINTS (OLD FORAAT) 2 DATA POINTS (OLO FORAAT) 1 DATA POINTS (OLD FORMAT) 7 DATA POINTS (OLO FORMAT) 10 DATA POINTS (OLO FORMAT) 1 DATA POINTS (OLO FORMAT) 19 DATA POINTS (OLO FORMAT) 15 DATA POINTS (OLO FORMAT) 73 DATA POINTS (OLO FORMAT)
STATISTICAL DISPLAY OF SEA LEVEL DATA:

GREECE, CRETE, ORMOS PROVIOES 44 DATA POINTS (OLO FORMAT) TURKEY, KAZIKBAGLAR PROVIDES 65 DATA POINTS (OLD FORMAT) ITALY. FORMIA LUNGOMARU PROVIDES 1 DATA POINTS (OLD FORMAT) ITALY, CAPE CIRCEO PROVIOES 1 DATA POINTS (OLD FORMAT) ITALY, ANZIO. ASTURA PROVIDES 1 OATA POINTS (OLD FORMAT) ITALY, CIVITAVECCHIA, PROVIOES 4 DATA POINTS (OLO FORMAT) ITALY, GIGLIO ISLANO, PROVIDES I OATA POINTS (OLO FORMAT) ITALY, ORBETELLO, COSA PROVIOES 2 DATA POINTS (OLO FORMAT) ITALY, LA SPEZIA, LUNI PROVIDES 2 DATA POINTS IOLD FORMAT: ITALY. ARGENTARIO, SANU PROVIOES 1 DATA POINTS (OLD FORMAT) ITALY, GIGLIO ISLAND, PROVIOES I DATA POINTS IOLD FORMATI BRD,NIEDERSACHSEN PROVIDES 24 UATA POINIS (INEW FORMAT) NL, ZEELAND PROVIDES 13 DATA POINTS (NEW FORMAT) AL.SOUTH-HOLLAND PROVIDES 21 UATA POINIS (NEL EORIMAI) NL.NORTH-HOLLAND PROVIDES 9 LATA POIIUTS INEW FURMAI) NL.GRONINGEN PROVIDES G DATA POINIS (NEW FORMAT) NL.FBIESLAND PROVIDES 1 LATA PUINIS (NEW FORMAI) NL, BRABANT PROVIDES 1 DATA POINTS (NEW FORMAI) STATEMENTS EXECUTED $=2608$ $5: 28.25 P M \quad 22$ AUG 78 HORST PREUSS

Abb. 6: Statistische Wiedergabe der Seespiegeldaten. 
Die Auswertung der Meeresspiegel-Basisdaten per Computer setzt voraus, daß ein ausgetestetes Programmsystem nicht nur zum Lesen und Auslisten der Daten, sondern auch für Prüfungen, Korrekturen, Angleichungen, Umrechnungen, Auswahl, Interpretationen und Darstellungen existiert. Die Entwicklung eines solchen Programmsystems wurde gegenüber der Datenaufnahme vorrangig behandelt. Die Zusammenarbeit mit dem Department of Geological Sciences, Cornell University, U.S.A., ergab die Möglichkeit einer Weiterentwicklung des im Konzept seit Mai 1978 fertigen Programmsystems L4SEA.

Das Programmsystem L4SEA besteht bis zum gegenwärtigen Zeitpunkt aus einem Hauptprogramm, das 4 Unterprogramme benutzt, die den graphischen Ausgang kontrollieren, und verschiedene kleinere Unterprogramme als graphische Grundbausteine für eingefügte Nummern, Symbole, Buchstaben usw. Die Abb. 1 zeigt ein Schema des Programmsystems zur Auswertung von Meeresspiegeldaten. Das Programm L4MEER zur Auslistung der Basisdaten ist mit eingefügt. Auf der linken Seite des Schemas steht die Eingabe der Daten per Lochkarte oder über Magnetband. Die rechte Seite zeigt den graphischen Ausgang und die möglichen Auslistungen (output on printer). Zwei Listentypen sind zu erwähnen:

a) Eine statistische Wiedergabe der Beobachtungspunkte, die den Namen des Landes bzw. des Staates oder den Meeressektor aus der oberen Zeile von Item 1 des Computerformblattes enthält und die Gesamtzahl der gespeicherten Daten aus dem spezifizierten Gebiet. Abb. 6 gibt ein Beispiel für eine solche Liste.

b) Eine Dokumentationsliste, die die Daten aller Items des Formblattes umsortiert nicht ausgewählt - wiedergibt (Abb. 4).

Der graphische Ausgang (output on plotter) ist in Form von 5 verschiedenen Typen von Zeit/Tiefen-Diagrammen vorgesehen, wovon 4 Diagrammtypen bis zum gegenwärtigen Zeitpunkt berechnet und gezeichnet werden können. Der Typ 5 ist noch in der Entwicklung. Der Aufbau der Diagramme ist einheitlich für alle Typen. Die Uberschrift beinhaltet die Angaben zum Land bzw. Staat oder Meeressektor, aus dem die Daten stammen, in der Form wie sie in Item 1 des Computer-Formblattes aufgeführt sind. Die Legende für die verschiedenen Typen erscheint unter dem Diagramm. Der Maßstab der Diagramme ist frei wählbar, wobei der Horizontalmaßstab unabhängig vom Vertikalmaßstab zu wählen ist (Abb. 7).

Der Diagrammtyp 1 (Abb. 7) gibt die Möglichkeit zur graphischen D a rstellu ng von unkorrigierten Probendaten. Jedes Datum ist durch ein Kästchen wiedergegeben, dessen Breite das Zeitintervall und dessen Höhe die Fehlerbreite der Tiefenbestimmung in Bezug zum Mittleren Meeresspiegel (= Tidemittelwasser) zeigt. Die Legende für Typ 1 gibt die Wasserspiegel-Indikation von 1 bis 9 an (entsprechend der Liste vom Formblatt) und erklärt die 5 Möglichkeiten eines geotektonischen Einflusses auf den Probenentnahme-Punkt. Diese werden durch kleine Pfeile angezeigt, die nach oben oder unten zeigen - entsprechend der Angabe im Formblatt.

Falls keine Fehlerintervalle im Formblatt angegeben sind, wird ein Modell zur Fehlerberechnung angewandt. Das Zeitintervall wird durch die Standard-Abweichung von \pm 1 Sigma angegeben. Alle Fehlerrechnungen sind nach der Gauss-Formel zur Berechnung des mittleren quadratischen Fehlers aus einer Anzahl von Einzelbeträgen ausgeführt, die eine schwache Vergrößerung des Gesamtfehlerintervalls mit einer ansteigenden Zahl von Fehlern zur Folge hat. Intervall- und Zentralpunktverschiebungen ergeben sich durch mögliche Modifikationen der im Diagramm des Typs 1 dargestellten Daten in folgenden Fällen: Wenn ein Zeitintervall angegeben ist, das das 1-Sigma-Intervall übersteigt, so wird dieses automatisch reduziert. Wenn in den Labordaten der $\mathrm{C}^{14}$-Altersbestimmung 
TIME-TQ-DEPTH DIAGRAM

SHOWING UNCORRECTED SAMPLE DATA FROM: U S A DE L A W A R E

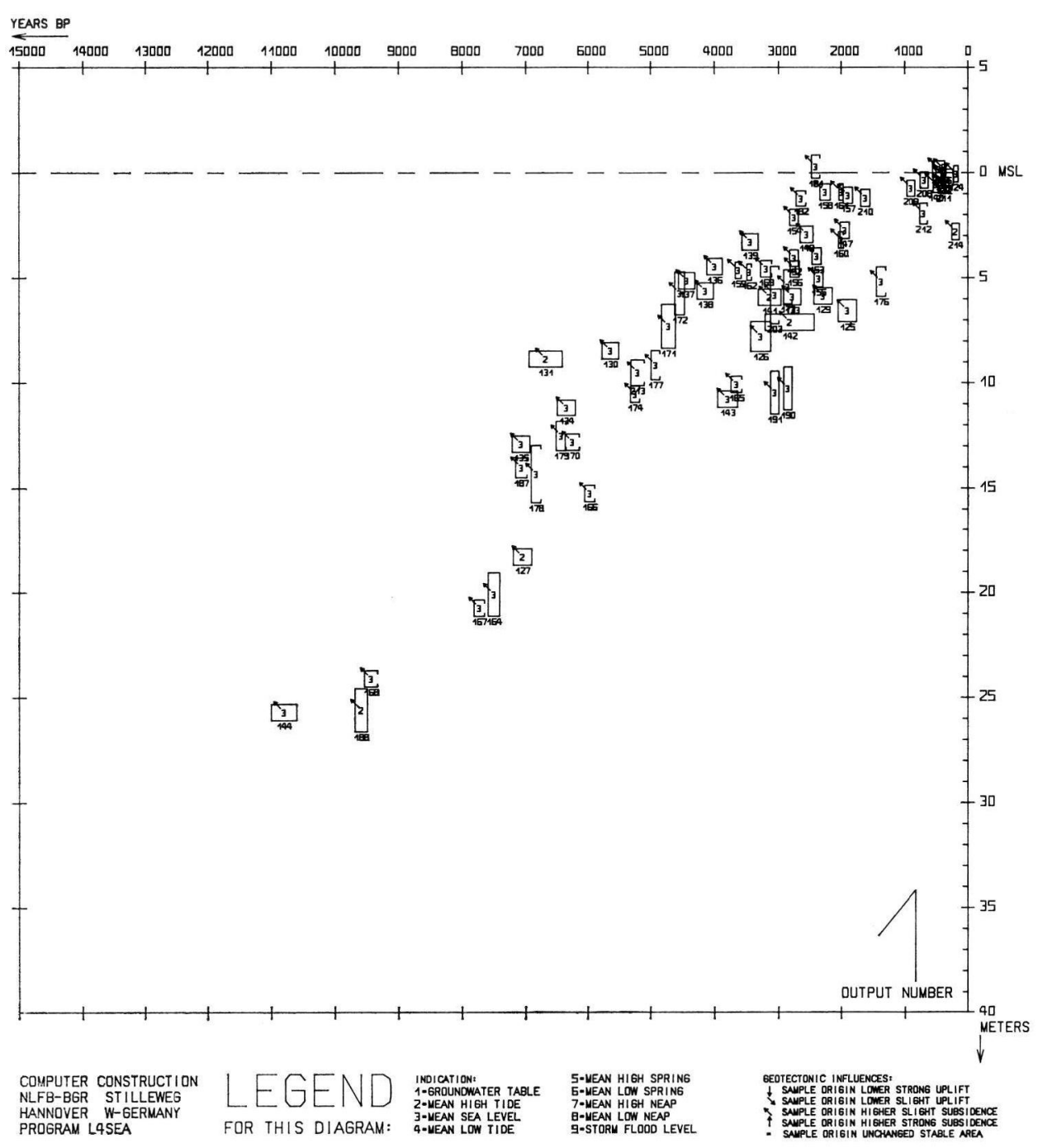

Abb. 7: Zeit/Tiefen-Diagramm des Typs 1 als Plotterausgabe.

eine Halbwertszeit des C14-Isotops angegeben ist, die nicht innerhalb der Werte von 5568 bis 5570 Jahren liegt, so werden die Alterswerte entsprechend einem Korrekturfaktor verschoben. Die im Formblatt angegebenen Korrekturen der C14-Alterswerte (z. B. SuEss-Korrektur, Masca-Korrektur usw.) beeinflussen bei unterschiedlicher Anwendung die Vergleichsmöglichkeit von Daten unterschiedlicher Einsender. Die Daten werden deshalb für die Darstellung in e in e m Diagramm auf eine einheitliche Berechnungsgrundlage zurückgebracht. 
Außer den hier genannten werden für Diagramme des Typs 1 keine weiteren Korrekturberechnungen ausgeführt. Korrekturen für geotektonische Einflüsse, Konsolidation/ Kompaktion, Meeresspiegel-Indikation usw. sind erst in den Berechnungen für die folgenden Diagrammtypen zu finden.

Diagramme des Typs 2 zur Darstellung von unkorrigierten Meeress pi e g e l-Indik a t or e n ergeben ein leicht abgeändertes Bild, besonders wenn einige Proben zur indirekten Datierung herangezogen worden sind. Die Kästchen sind dann entsprechend dem Alter und der Tiefe des Wasserspiegel-Indikators verschoben. Korrek-
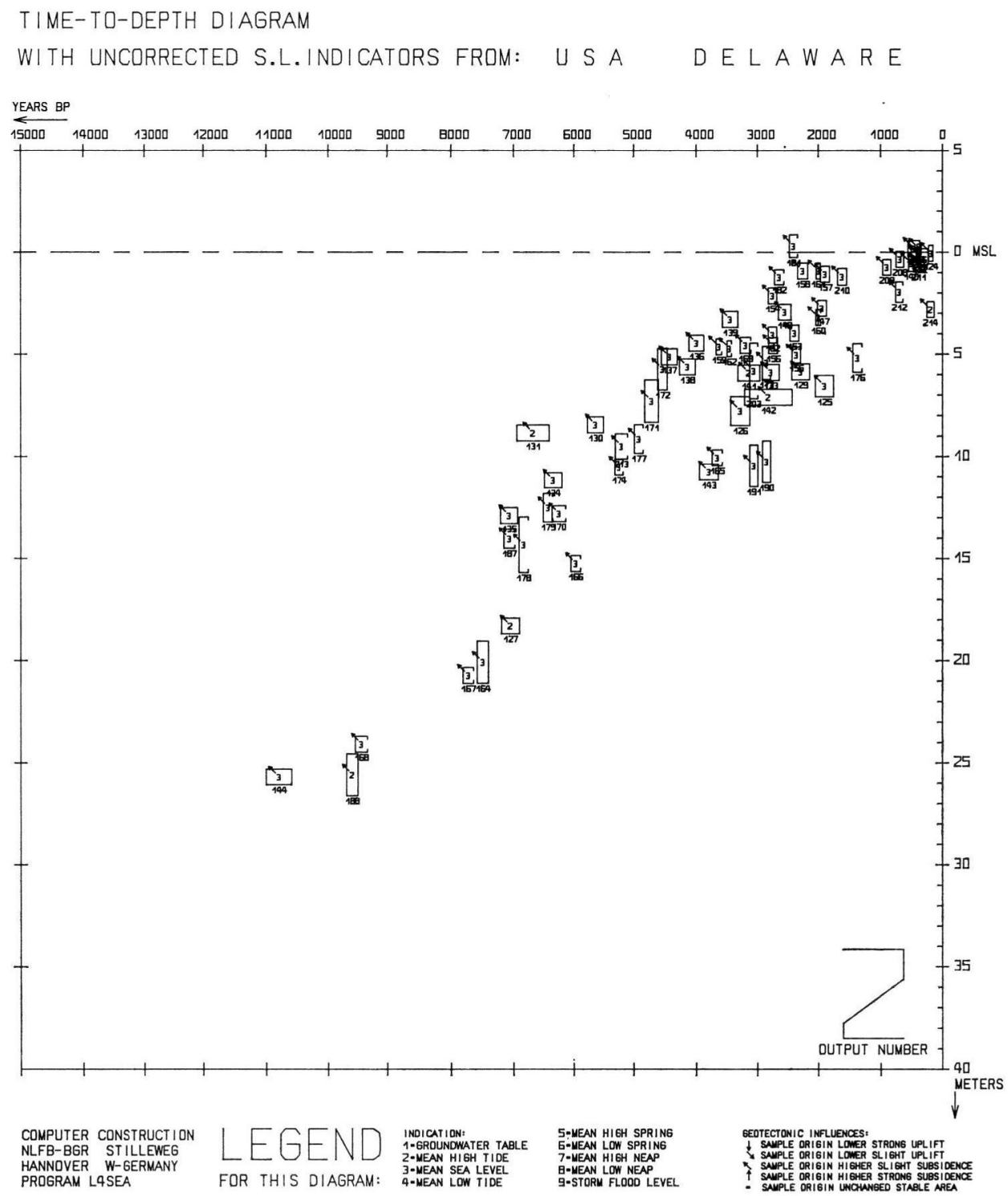

Abb. 8: Zeit/Tiefen-Diagramm des Typs 2 als Plotterausgabe. 
turen in Bezug auf Kompaktion/Konsolidation, geotektonische Einflüsse usw. sind jedoch auch hier noch nicht errechnet und angewendet. Lediglich die Tiefenintervalle der Probendaten sind gegenüber den Diagrammen des Typs 1 neu berechnet. Für spezielle Indikationen ergibt sich ein vergrößertes Intervall, denn außer den Fehlerbereichen, die sich aus Messungsungenauigkeiten bei der Nivellierung und Festlegung der Marke des Mittleren Meeresspiegels ergeben, wird hier auch der indikative Bereich (= indicative range) des Meeresspiegel-Indikators mit in die Fehlerberechnung einbezogen. Falls keine Angaben zum indikativen Bereich im Computer-Formblatt gegeben sind, wird ein Modell zur Errechnung dieses Bereiches angewandt. Dieses Modell arbeitet bislang recht befriedigend für die verschiedenen Torfarten. Hier soll ein Beispiel gegeben werden:

Es sei eine Probe aus einer Lage von Phragmites-Torf entnommen. Das Probenintervall beträgt $10 \mathrm{~cm}$. Der Meßfehler bei der Höheneinmessung auf NN beträgt $\pm 7 \mathrm{~cm}$. Die Bestimmung des Mittleren Meeresspiegels (= Tidemittelwasser) in Bezug auf NN verursacht einen zusätzlichen Fehler von $\pm 5 \mathrm{~cm}$. Das ergibt ein Tiefenintervall $\mathrm{A}=\sqrt{10^{2}+(2 \cdot 7)^{2}+(2 \cdot 5)^{2}} \approx 20 \mathrm{~cm}$ für die Darstellung im Diagramm des Typs 1. Der indikative Bereich des Wasserspiegel-Indikators „Phragmites-Torf“ ist jedoch nach Untersuchungen von SCHEER (1953) größer. ScheER nennt ein Intervall von -26 bis $+72 \mathrm{~cm}$ bezogen auf MThw, also insgesamt $98 \mathrm{~cm}$ für das Vorkommen von Phragmites communis. Wenn wir von einer Pflanzendichte ausgehen, die zur Torfbildung führte, so kann für Phragmites-Torf - in Anlehnung an die Gauss-Normalverteilungskurve ein um ca. 30\% kleineres Intervall angenommen werden. Es liegt bei ca. $70 \mathrm{~cm}$. Die Addition ergibt ein Tiefenintervall $\mathrm{B}=\sqrt{20^{2}+70^{2}} \approx 73 \mathrm{~cm}$ für die Darstellung im Diagramm des Typs 2 (Abb. 8).

Die Wachstums-Intervalle für Pflanzen anderer Torfarten sind dem Computer ebenfalls [in Form einer DATA-Liste] mitgeteilt. Schwierigkeiten gibt es jedoch noch mit der Umrechnung von Grundwasseranzeigern auf das Niveau des Mittleren Meeresspiegels für die Darstellung im Diagramm des nächsten Typs.

Diagramme des Typs 3 zeigen korrigierte Meeresspiegel-Indikatoren und ihre Zeit- und Tiefenintervalle. Hier sind Korrekturwerte für geotektonische Einflüsse errechnet worden, sofern Angaben dazu im Formblatt enthalten sind. Falls keine Beträge oder Schätzwerte gegeben sind, jedoch eine Absenkung bzw. eine Landhebung durch Vorzeichen angezeigt ist, kann ein Datensatz mit einem Korrekturwert belegt und umgerechnet werden. Dazu ist die Eingabe eines Schätzwertes über die Parameterkarte (Abb. 1) notwendig. Der Schätzwert wird als Gesamtbetrag der geotektonischen Einflüsse pro Zeiteinheit - gültig für ein ausgesuchtes Gebiet - gewählt. Dadurch wird dem Benutzer des Programmsystems die Möglichkeit gegeben, Diagrammkonstellationen nach seinen Wünschen zu erzeugen, bis eine Konstellation erreicht ist, die derjenigen von stabilen Gebieten entspricht. Der jeweilige Schätzwert muß dann festgehalten werden.

Hier können natürlich auch errechnete Werte bestehender Modelle ${ }^{1}$ ) eingegeben werden, und interessante Vergleichsmöglichkeiten sind gegeben. Aber, falls mehrere Faktoren neben der eustatischen Komponente an den relativen Schwankungen des Meeresspiegels im ausgewählten Gebiet beteiligt sind, so besteht bislang keine Möglichkeit einer Trennung. Die Programme für die Faktoranalyse sind erst in der Entwicklung.

Die Korrektur für Konsolidation/Kompaktion basiert auf den im Computer-Formblatt angegebenen Werten. Diese sind meist aus Vergleichen mit dicht benachbarten Beobachtungspunkten abgeleitet. Falls keine Werte gegeben sind, wird eine Modellrechnung angewandt. Hierbei wird die mögliche Kompaktion aus der Tiefe der Probe unter der heutigen Oberfläche berechnet und das Probenmaterial sowie hangende und liegende Se-

1) z. B. das Modell für Eis- und Hydroisostasie von ClaRK et al. (1978). 
dimente berücksichtigt. Außerdem geht der Zeitfaktor mit in die Berechnung ein. Da die Zahl der möglichen Fehler mit zunehmender Zahl der Einzelberechnungen steigt, muß zusätzlich das Tiefenfehlerintervall vergrößert werden. Erosion und eventuelle Umlagerung der Probe mögen bei entsprechend gekennzeichneten Daten ernste Fehler verursachen, die größer sind als die dargestellten Fehlerintervalle. Um dieses und vermutete Kontamination anzuzeigen, sind besondere Darstellungen der Kästchen programmiert worden. Die beeinflußte Seite des Kästchens wird offen gelassen. Offene Kästchen zeigen also Meeresspiegel-Indikatoren, die unter dem Einfluß möglicher intervallüberschreitender Tiefen- und/oder Zeitfehler stehen (Abb. 9).

\section{TIME-TO-DEPTH DIAGRAM}

\section{SHOWING CORRECTED S.L.INDICATORS FROM: US A DE L A W A R E}

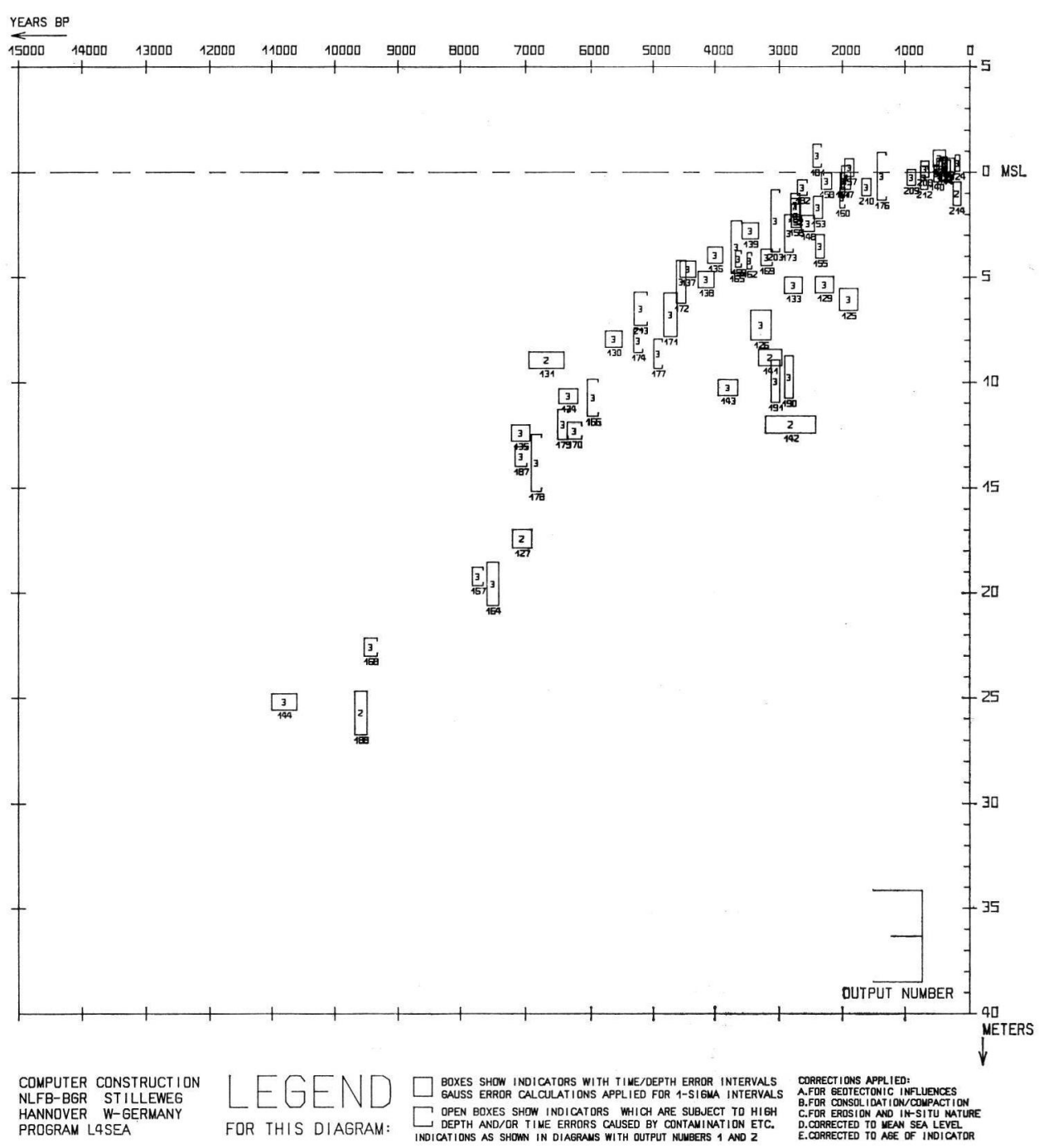

Abb. 9: Zeit/Tiefen-Diagramm des Typs 3 als Plotterausgabe. 
Die Umrechnung der Höhenlage des Meeresspiegel-Indikators auf das Niveau des Mittleren Meeresspiegels geschieht aufgrund der Informationen zur Höhe der Probe und zum Paläotidenhub. Falls der Betrag des fossilen Tidenhubs unbekannt ist, wird ein Schätzwert aus dem Betrag des heutigen Tidenhubs abgeleitet. Das geschieht durch leichte Modifikation in Abhängigkeit von den Angaben zur (Paläo)geographie. Falls Sturmflutniveaus angezeigt sind, werden die entsprechenden Kästchen nach oben verschoben, und zwar über die Hochflutmarke. Außerdem wird dann das Höhenfehlerintervall vergrößert.

Falls ein Grundwasserspiegel angezeigt ist, wird ein Modell vom Grundwasserfluß angewendet. Dieses Modell ist jedoch unvollkommen und eine der größten Fehlerquellen. Die Entfernung des Probenentnahmepunktes zur heutigen Küstenlinie, die Konfiguration der heutigen Küstenlinie und die Konfiguration der fossilen Küstenlinie sind zwar meistens bekannt, die Entfernung zur fossilen Küstenlinie und die Neigung des fossilen Grundwasserspiegels dagegen unbekannt. Näherungswerte lassen sich nur innerhalb großer Fehlerbreiten errechnen. Hier gehen die Angaben zum Typ des Indikators, zum beprobten Material und zum liegenden Material mit in die Rechnung ein.

Es hat sich außerdem als notwendig erwiesen, die Angaben zum angezeigten Wasserspiegel intern zu überprüfen und die Indikation mit den Angaben zum beprobten Material zu vergleichen. Viele Einsender sind sich der Indikation ihrer Proben nicht bewußt und so kommen unterschiedliche Angaben für gleiches Material in gleicher Sedimentabfolge zustande. Um die Verwirrung über die Indikation von Torflagen innerhalb subaquatisch gebildeter klastischer Sedimente zu lösen, waren mehrere Computertestläufe mit echten Daten notwendig. Das Ergebnis war, daß Phragmitestorf als sedentäre Bildung eines fossilen Küstensumpfes im Tidebereich kein Anzeiger für den Mittleren Meeresspiegel ist, sondern einen Wasserspiegel nahe dem Mitteltidehoch wasser anzeigt (siehe auch SCHEER 1953).

Die übrigen Niedermoortorfarten sind Anzeiger für einen zeitgleichen Grundwasserspiegel, wobei der indikative Bereich von Torfart zu Torfart unterschiedlich ist.

Ein Beispiel für Diagramme des Typs 3 ist die Abbildung 9. Es zeigt korrigierte Meeresspiegel-Indikatoren aus dem Gebiet Delaware (USA). Der Vergleich von Abb. 7 (Diagramm des Typs 1) mit Abb. 9 (Diagramm des Typs 3) zeigt, daß die Korrekturen sehr effektiv waren. Die Anordnung der einzelnen Datenkästchen im Diagramm des Typs 3 ist nach Anwendung aller Korrekturmodelle derart klar, daß sich leicht die beiden einschließenden Kurven konstruieren lassen, zwischen denen der weitaus größte Teil der Kästchen liegt. Die Abbildung 10 gibt ein Beispiel für die Handkonstruktion der Einschlußkurven. Einige „Ausreißerdaten“ sind deutlich erkennbar. Große Fehlerintervalle zeigen in vielen Fällen deren geringe Qualität.

Die zu ermittelnde Meeresspiegel-Anstiegskurve ist in dem Bereich zwischen den beiden Einschlußkurven zu erwarten. Da jedoch der Fehlerbereich (zwischen den Kurven) in diesen Beispielen eine Schwankungsbreite von 2 bis $4 \mathrm{~m}$ einnimmt, werden alle tatsächlich vorkommenden Meeresspiegel-Schwankungen dieses Ausmaßes vom Fehlerbereich verschluckt. Eine undulierte Meeresspiegelkurve läßt sich deshalb nicht ohne Zusatzinformation konstruieren.

Die Diagramme des Typs 4 werden mit dem Ziel gezeichnet, K onstruktionshilfen für Schwankungskurven innerhalb der Darstellung korrigierter Meeresspiegel-Indikatoren zu geben. Sie stellen korrigierte Meeresspiegel-Indikatoren in Kästchenform dar, wobei jedem Kästchen Zusatzzeichen beigegeben werden. Diese zeigen die Tendenzen der Sedimentation zur repräsentierten Zeit als Großbuchstaben im Kästchen. Der Buchstabe „R“ steht für regressive Überlagerung, „ $\mathrm{T}$ “ für transgressive Überlagerung. Beides läßt sich aus der Position der Probe im 
Profil und speziell bei Torfproben aus der Position der Probe in der Torflage ableiten. $\mathrm{Da}$ Sedimentationstendenzen allein keine Aussagekraft in Bezug auf Meeresspiegelschwankungen besitzen, werden zusätzliche Informationen aus dem beprobten Profil ausgewertet und dargestellt, z. B. der obere und der untere Sedimentkontakt zum Meeresspiegel-Indikator. Die Abfolge von Fazieseinheiten ist im Formblatt nach abnehmendem marinen Einfluß geordnet (Charakter 1 bis 9), so daß durch Differenzbildung, die leicht zu erreichen ist, für jede Probe Positiv- oder Negativwerte des marinen Einflusses zur Verfügung gestellt werden können. Diese Werte werden in Form von auf- bzw. abzeigenden Pfeilen

\section{TIME-TO-DEPTH DIAGRAM}

\section{SHOWING CORRECTED S.L.INDICATORS FROM: U S A DE L A W A R}

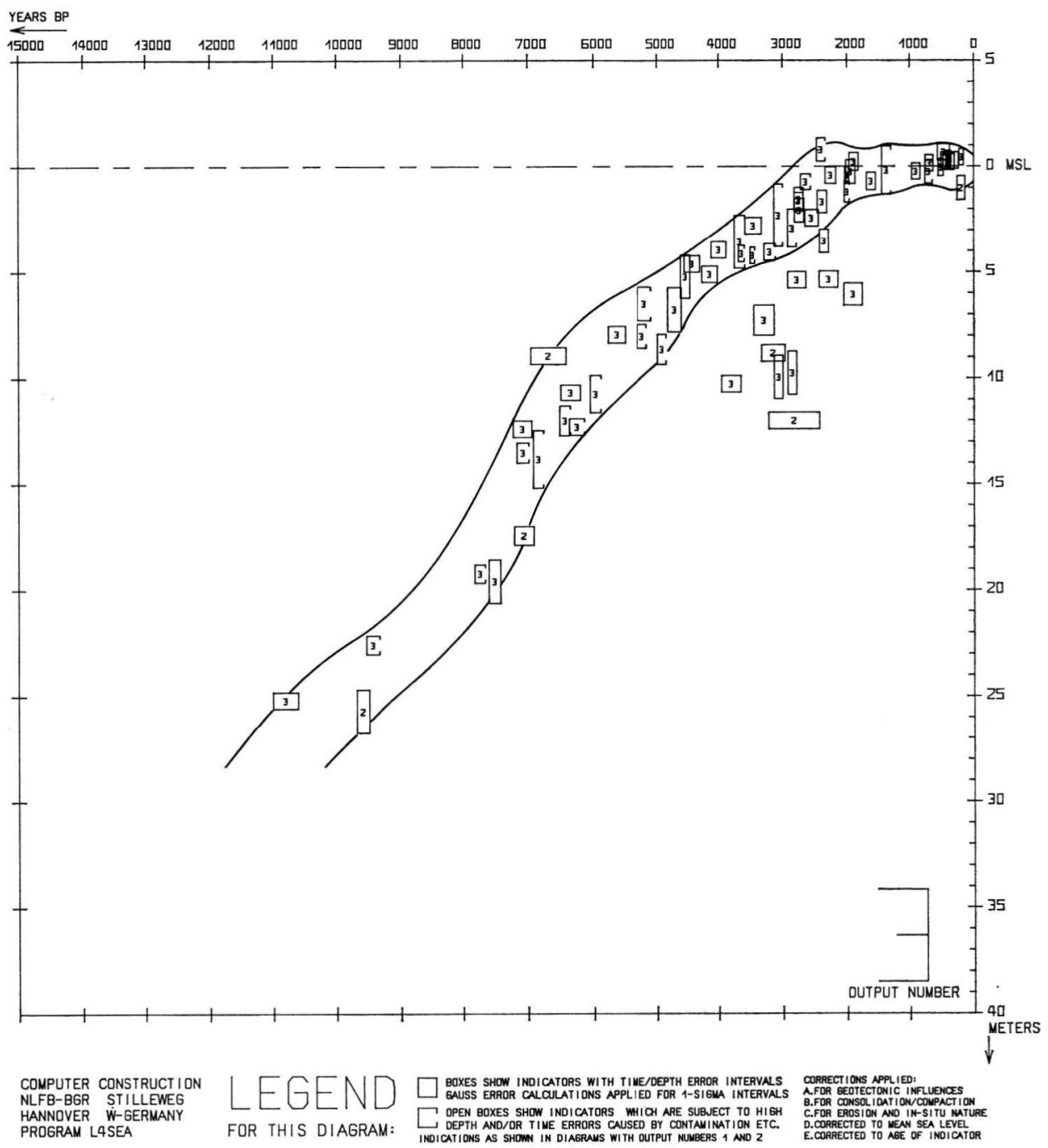

Abb. 10: Zeit/Tiefen-Diagramm des Typs 3 mit Einschlußkurven. 
TIME-TO-DEPTH DIAGRAM

SHOWING CORRECTED S.L.INDICATORS FROM: T K Z Z 17 . N O R D H L L Z

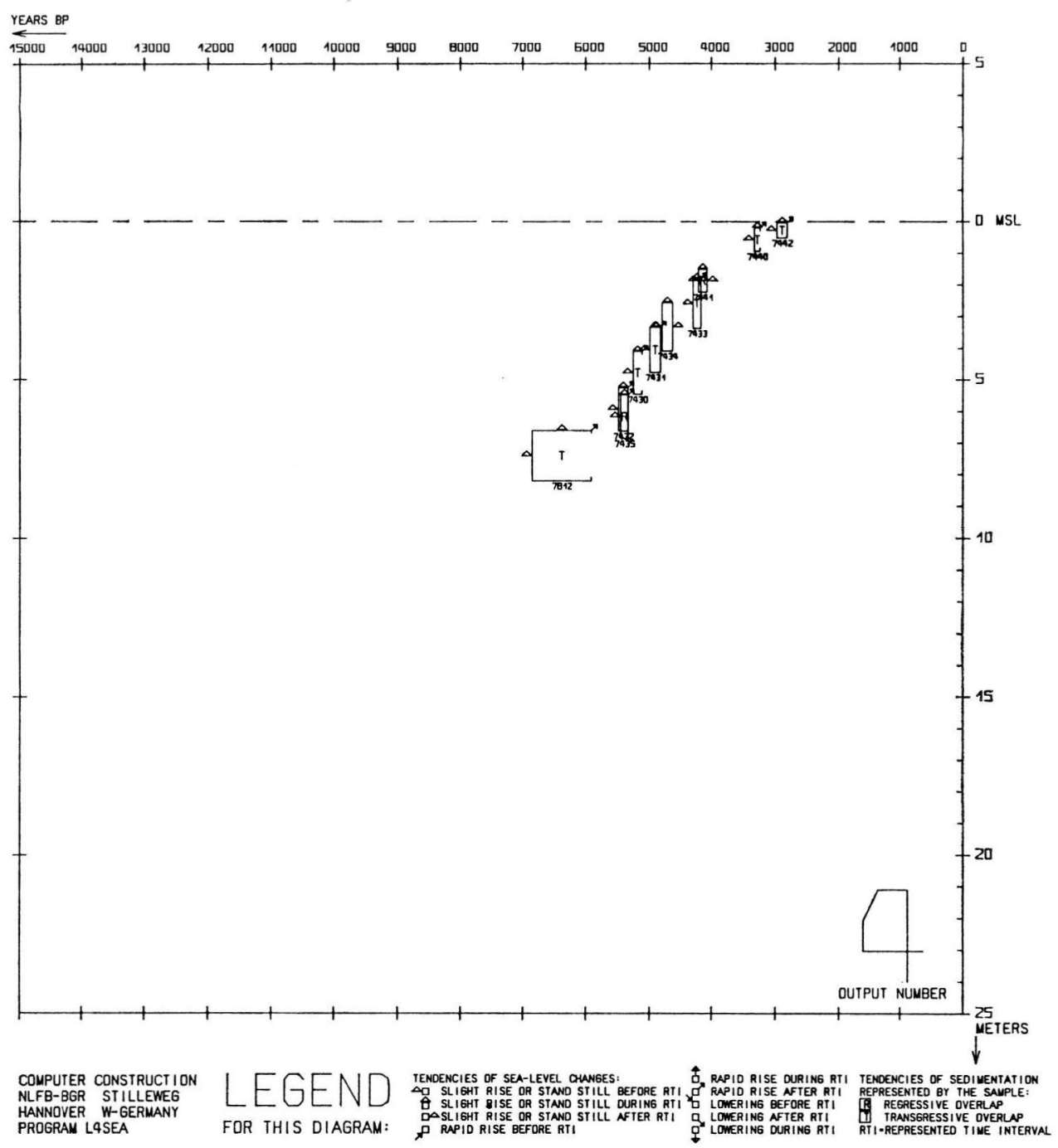

Abb. 11: Zeit/Tiefen-Diagramm des Typs 4.

dargestellt. In der Auswertung gibt die Häufung gleicher Tendenzen im gleichen Zeitintervall Anhaltspunkte für die Konstruktion undulierter Meeresspiegel-Schwankungskurven. Je größer das Beobachtungsgebiet durch eine entsprechende Einbeziehung größerer Datenmengen in die Berechnungen wird, um so deutlicher müßten überregional gleichlaufende Tendenzen werden. Diese Auswertungsphase leitet schon zur - noch nicht programmierten - Faktoranalyse über. Die Abb. 11 gibt ein Beispiel für den Diagrammtyp 4. 
Diagramme des Typs 5 lassen sich noch nicht berechnen und darstellen, da die Konstruktion "glatter Kurven“ eines speziellen Programmes bedarf, das z. Z. noch in der Testphase ist. Vorgesehen sind die Darstellung der beiden Einschlußkurven und die Schraffur der eingeschlossenen Fläche.

\section{Schriftenverzeichnis}

Clark, J. A., Farrel, W. E. \& Peltier, W. R. (1978): Global changes in postglacial sea level: a numerical calculation, Quaternary Res. 9: 265-287.

Scheer, K. (1953): Die Bedeutung von Phragmites communis Trin. für die Fragen der Küstenbildung, Probleme der Küstenforschung im Geb. d. südl. Nordsee, 5: 15-25; Hildesheim.

Anmerkung zu den Abbildungen:

Die Abbildungen 7, 8, 9 und 10 enthalten Daten, die gesammelt und publiziert worden sind von:

Kraft, J. C. (1976): Radiocarbon dates in the Delaware coastal zone (eastern Atlantic coast of North America) - Delaware Sea Grant Techn. Rep., DEL-SG-1976, University of Delaware, 20 p.; Newark.

Die Abbildungen 5 und 11 enthalten nicht-publizierte, vom Autor gesammelte Meeresspiegeldaten.

Manuskript eingegangen am 12. 2. 1980. 
